Service social

\title{
Le regard de jeunes pères sur leur enfance et leur adolescence
}

\section{Jean-Martin Deslauriers}

Volume 58, numéro 1, 2012

URI : https://id.erudit.org/iderudit/1010437ar

DOI : https://doi.org/10.7202/1010437ar

Aller au sommaire du numéro

Éditeur(s)

École de service social de l’Université Laval

ISSN

1708-1734 (numérique)

Découvrir la revue

Citer cet article

Deslauriers, J.-M. (2012). Le regard de jeunes pères sur leur enfance et leur adolescence. Service social, 58(1), 12-31. https://doi.org/10.7202/1010437ar

\section{Résumé de l'article}

Cet article présente un volet des résultats d'une recherche menée à Gatineau auprès de 30 jeunes pères ayant eu un enfant avec une jeune femme de moins de 20 ans. Nous avons voulu connaître leur point de vue sur leur enfance, leur adolescence, jusqu'à leur expérience de la paternité pour situer celle-ci dans leur trajectoire de vie. Les résultats indiquent quatre tendances principales, décrivant la façon dont l'enfance et l'adolescence furent vécues : heureuse, " correcte », difficile et en situation de survie. L’adolescence fut une période trouble pour presque tous les jeunes hommes, bien qu'une majorité ait estimé avoir connu une enfance heureuse. Presque tous les jeunes hommes (27 sur 30) ont présenté au moins un problème de comportement parmi les quatre suivants : expulsion de l'école, dépendance à une substance, batailles à l'école ou tentative de suicide. Ceux qui estiment avoir connu une enfance heureuse présentent plus de persévérance scolaire que ceux qui déclarent avoir vécu une enfance difficile ou traumatisante. Les premiers sont aussi beaucoup plus nombreux à avoir bénéficié d'une vie relativement stable. Néanmoins, leurs rapports avec les adultes en autorité et avec les structures sociales ont souvent été tendus. Également, ces résultats indiquent que l'expérience de l'enfance n'est pas directement liée à l'engagement paternel des jeunes pères. Par exemple, ceux qui ont connu des enfances difficiles sont très présents dans la vie de leur enfant à la dernière cueillette de données.
Ce document est protégé par la loi sur le droit d'auteur. L’utilisation des services d'Érudit (y compris la reproduction) est assujettie à sa politique d'utilisation que vous pouvez consulter en ligne.

https://apropos.erudit.org/fr/usagers/politique-dutilisation/ 


\section{Le regard de jeunes pères sur leur enfance et leur adolescence}

\section{Jean-Martin Deslauriers}

Cet article présente un volet des résultats d'une recherche menée à Gatineau auprès de 30 jeunes pères ayant eu un enfant avec une jeune femme de moins de 20 ans. Nous avons voulu connaître leur point de vue sur leur enfance, leur adolescence, jusqu'à leur expérience de la paternité pour situer celle-ci dans leur trajectoire de vie. Les résultats indiquent quatre tendances principales, décrivant la façon dont l'enfance et l'adolescence furent vécues: heureuse, « correcte », difficile et en situation de survie. L'adolescence fut une période trouble pour presque tous les jeunes hommes, bien qu'une majorité ait estimé avoir connu une enfance heureuse. Presque tous les jeunes hommes (27 sur 30) ont présenté au moins un problème de comportement parmi les quatre suivants : expulsion de l'école, dépendance à une substance, batailles à l'école ou tentative de suicide. Ceux qui estiment avoir connu une enfance heureuse présentent plus de persévérance scolaire que ceux qui déclarent avoir vécu une enfance difficile ou traumatisante. Les premiers sont aussi beaucoup plus nombreux à avoir bénéficié d'une vie relativement stable. Néanmoins, leurs rapports avec les adultes en autorité et avec les structures sociales ont souvent été tendus. Également, ces résultats indiquent que l'expérience de l'enfance n'est pas directement liée à l'engagement paternel des jeunes pères. Par exemple, ceux qui ont connu des enfances difficiles sont très présents dans la vie de leur enfant à la dernière cueillette de données.

Mots-clés : Jeune père, enfance, adolescence, transition familiale, engagement paternel, persévérance scolaire

\section{RECENSION DES ÉCRITS}

On connaît très peu le point de vue des jeunes pères sur leur propre vie, notamment sur leur passé familial au cours de l'enfance et de l'adolescence (Tuffin, Rouch et Frewin, 2010). Les écrits tendent à démontrer que, tant sur le plan psychosocial que familial et socioéconomique, les jeunes pères ont un profil de vulnérabilité très semblable à celui des jeunes mères (Goulet et al., 2001).

La culture héritée de la famille influence les comportements des jeunes dans leurs rapports amoureux et par la suite, leur façon de devenir pères. Les jeunes pères ont plus souvent que les autres jeunes connu des milieux familiaux difficiles (Furstenberg et Weiss, 2000). Beaucoup plus fréquemment que la population de jeunes hommes sans enfant du même groupe d'âge, ils ont subi des abus physiques ou sexuels ou ont été témoins de violence conjugale (Anda, Felitti, Chapman et al., 2001), ont souffert de l'absence de leur père, de son désengagement ou de rapports conflictuels avec celui-ci (Allen et Doherty, 1996; Tuffin, Rouch et Frewin, 2010).

Les jeunes pères proviennent souvent d'une famille de statut socioéconomique bas (Kiernan, 1992; Furstenberg et Weiss, 2000), dont les parents eurent des comportements violents (Kiernan, 1992; Sigle-Rushton, 2005; Dearden, Hale et Blankson, 1994), sont pauvres 
(Dearden, Hale et Woolley, 1998) et demeurent dans un quartier présentant un taux élevé de délinquance juvénile (Jaffee, Caspi, Moffitt, Taylor et Dickson, 2001; Pears et al., 2005). L'étude de Thornberry, Smith et Howard (1997), confirmée par d'autres (Stouthamer-Loeber et Wei, 1998; Capaldi, Pears, Patterson et Owen, 2003), montre aussi que d'être jeune délinquant augmente la probabilité de devenir père de façon précoce. Un bon nombre des jeunes pères participant à ces recherches étaient des adolescents présentant des problèmes de comportement (Dearden, Hale et Alvarez, 1992; Kiernan, 1992; Hanson, Morrison et Ginsburg, 1989). Les comportements antisociaux de différents ordres, comme la consommation de substances psychoactives ou des comportements sexuels à risque, sont souvent observés chez les adolescents avant qu'ils ne deviennent pères (Pears et al., 2005). On pourrait en conclure que les jeunes qui ont des comportements à risque et qui proviennent de milieux socioéconomiques défavorisés ont plus de chances que les autres de devenir pères avant l'âge adulte (Kiselica, 2008).

Enfin, le milieu familial du jeune père présente une plus grande acceptation de la monoparentalité (Furstenberg et Weiss, 2000). En effet, plus fréquemment que chez la population de jeunes dans son ensemble, les jeunes pères vont souvent trouver dans leur famille immédiate des modèles de paternité ou de maternité précoce (Jaffee et al., 2001). D'ailleurs, les jeunes parents sont plus souvent nés de mères adolescentes et ressentent moins de jugement envers cette situation familiale que ceux qui sont nés alors que leurs parents étaient plus vieux (Allen et Doherty, 1996).

Concernant leur propre expérience de parentalité, la désunion guette les couples adolescents. Selon une étude, le quart des couples âgés de moins de 20 ans sont déjà désunis durant la grossesse, ce qui laisse entrevoir un taux important de séparations par la suite (Charbonneau, 1999). De plus, des chercheurs mentionnent que les jeunes hommes qui deviennent pères manifestent souvent peu de préoccupation par rapport à leur relation amoureuse, ne la perçoivent pas comme un projet à long terme et se montrent peu habiles en résolution de conflits (Allen et Doherty, 1996).

Cela dit, la vulnérabilité du couple rend d'autant plus nécessaire et crucial le développement d'un lien père-enfant dès le départ. À la différence des jeunes mères, plusieurs jeunes pères ne vivront pas avec leur enfant et ne le soutiendront pas financièrement (Hardy et Zabin, 1991). Par contre, d'autres sont très préoccupés par le bien-être de leur progéniture (Reeves, 2006).

Quoique plusieurs écrits mettent l'accent sur les difficultés des jeunes pères, d'autres nuancent ces données en faisant ressortir leur volonté et leur désir d'améliorer leur vie et de bien exercer leur rôle parental (Ouellet, Milcent et Devault, 2006; Thompson et Walker, 2004). En effet, les jeunes hommes qui ont connu des conduites marginales à l'adolescence peuvent avoir le désir de s'intégrer sur le plan socioéconomique afin de s'acquitter des responsabilités liées à leur nouveau rôle de père (Deslauriers, 2010; Frewin, Tuffin et Rouch, 2007). De fait, le désir des jeunes pères d'être présents pour leur enfant est un thème récurrent dans les résultats de plusieurs recherches (Featherstone, 2009). 


\section{MÉTHODOLOGIE}

\section{La cueillette de données}

Le recrutement de futurs jeunes pères s'est effectué sur deux ans au Centre local de services communautaires (CLSC) de Gatineau, dans le cadre des Services intégrés en périnatalité et pour la petite enfance à l'intention des familles vivant en contexte de vulnérabilité (Ministère de la santé et des services sociaux du Québec, 2004). Trente participants ont été recrutés dans le contexte de services prénataux et postnataux sur la base de l'intégration de jeunes mères au programme. Peu de temps après l'annonce de la grossesse (Temps 1), 17 des 30 participants ont été interrogés. Un mois avant la naissance de leur enfant (Temps 2), ce chiffre était de 15 participants, deux mois après la naissance de leur enfant (Temps 3 ), de 17 participants, et près d'un an après la naissance de leur enfant (Temps 4), de 26 participants. Un total de 77 entrevues furent réalisées, soit un peu plus de deux entrevues et demie en moyenne par jeune père. Tous n'ont pu être rencontrés à tous ces temps de mesure pour des raisons variées, telles que des difficultés à joindre les jeunes hommes, l'annulation d'entrevues et leurs horaires de travail.

Durant la phase prénatale (T1 et T2), les entrevues ont porté sur certains aspects de leur vie: enfance, adolescence, caractéristiques individuelles, vie amoureuse, grossesse, conception de la paternité, soutien de l'entourage et perception de l'avenir. Les entrevues étaient semi-structurées, laissant aux jeunes hommes la latitude d'aborder les différents aspects à leur rythme et sous l'angle qui leur convenait. Malgré cette souplesse dans la conduite des entrevues, l'enfance et l'adolescence ont toujours été abordées. L'objectif étant surtout de dégager une perspective globale de leur trajectoire de vie, nous n'avons toutefois pas cherché à aborder systématiquement des aspects particuliers, tels que leur consommation de substances, la violence de leurs parents ou leurs comportements à risque.

Pour ce qui est de la phase postnatale (T3 et T4), les entrevues ont porté surtout sur leur expérience de la paternité, leurs croyances et leurs perceptions face à leur rôle parental, l'expérience émotive qui s'y rattachait, avant et après la grossesse (par ex. : l'importance attribuée à son rôle auprès de l'enfant, le sentiment de compétence, les inquiétudes, etc.). Enfin, des données sociodémographiques furent recueillies à partir d'un questionnaire. Elles comprenaient quelques données sur le milieu familial d'origine du jeune père (voir le tableau I).

Le présent article rapporte essentiellement les données tirées de la première entrevue effectuée auprès de chacun des participants, durant laquelle un survol de leur histoire de vie était effectué. Une fois réalisée cette discussion sur leur passé, nous n'y revenions pas lors des entrevues subséquentes. 
TABLEAU I

Données sociodémographiques concernant l'enfance et l'adolescence

\begin{tabular}{|c|c|c|}
\hline Variable & Moyenne & Écart type \\
\hline \multicolumn{3}{|l|}{ Famille d'origine } \\
\hline Conjointes des grands-pères connues par les participants & 1,8 & 2,25 \\
\hline Conjoints des grands-mères connus par les participants & 4 & 4,2 \\
\hline Habité dans une famille d'accueil & 2 & 0,35 \\
\hline (Si oui) Nombre de familles d'accueil & 3,5 & 2,65 \\
\hline Nombre de déménagements & 7,3 & 5,97 \\
\hline \multicolumn{3}{|l|}{ Histoire personnelle à l'adolescence } \\
\hline Âge à la première relation sexuelle & $\begin{array}{c}15 \text { ans et } \\
1 \text { mois }\end{array}$ & 2,03 \\
\hline Nombre de partenaires sexuelles incluant leur partenaire & 7,7 & 10,12 \\
\hline Avortements connus (anciennes compagnes et/ou partenaire) & $33 \%$ & N/A \\
\hline Nombre d'enfants & 1,07 & 0,25 \\
\hline
\end{tabular}

Également, les questionnaires sociodémographiques ont permis de documenter les conditions socioéconomiques et familiales qui existaient au moment de l'annonce de la grossesse (T1) et un an après la naissance de leur enfant (T4). Ces résultats sont présentés dans le tableau II.

TABLEAU II

\section{Quelques indicateurs liés au passage vers la paternité}

\begin{tabular}{|c|}
\hline 30 jeunes pères ayant participé à la recherche : \\
\hline - Moyenne d'âge : 19 ans \\
\hline - Présents à la naissance de leur enfant : 28 \\
\hline - Ont signé l'acte de naissance : 29 \\
\hline - En couple un an après la naissance : 22 \\
\hline - Avaient des contacts réguliers avec leur enfant alors que celui-ci était âgé de 1 an : 29 \\
\hline $\begin{array}{l}\text { - Revenu augmente de } 33 \% \text { entre l'annonce de la grossesse (1187 \$/mois) et le moment où leur } \\
\text { enfant atteint un an (1661 \$/mois) }\end{array}$ \\
\hline - Taux de cohabitation augmente, particulièrement en appartement : $(\mathrm{T} 1=8 ; \mathrm{T} 4$ = 18) \\
\hline
\end{tabular}




\section{L'analyse des données}

Le codage des données à l'aide du logiciel NVIVO a permis de regrouper les extraits d'entrevues par catégories, notamment en ce qui concerne les caractéristiques des jeunes pères, leur vie amoureuse avec la mère de leur enfant, leurs perceptions par rapport à la paternité et le soutien dont ils bénéficient. Dans le cadre de cet article, les catégories présentées et analysées sont celles qui regroupent les propos des participants sur leur enfance, sur leur adolescence et sur l'influence d'expériences vécues à ces étapes sur leur passage vers la paternité. À partir de la méthode de théorisation ancrée, de l'étude de chaque trajectoire de vie a émergé une typologie de quatre sortes de perceptions exprimées par les jeunes pères. Trouver des titres à ces catégories posa un certain défi, en ce qui concerne les trajectoires qui comportaient plus de moments trouvés difficiles et celles qui comportaient de la violence familiale. II fut plus facile de partir de leurs mots pour définir des enfances « heureuses » ou « correctes », car c'est ainsi que plusieurs les définissaient d'emblée. Par contre, pour les deux autres catégories, les termes d'enfances « difficiles » ou « de survie » furent tirés du langage courant pour désigner un genre d'expériences. II s'agissait de trouver des concepts pour distinguer et regrouper des propos allant dans le même sens, et non pas d'un jugement.

Au cours de l'organisation des résultats, nous avons dû composer avec le manque de cohérence interne que présentaient certains propos. Par exemple, certains estimaient que leur enfance était heureuse, alors que par ailleurs ils décrivaient beaucoup de moments difficiles. D'autres participants tendaient à banaliser ces expériences difficiles. Ces incohérences ont nécessité de faire un choix méthodologique entre les résultats bruts et leur interprétation par les participants : nous avons choisi de préserver le point de vue de ceux-ci en classant leurs propos selon les qualificatifs qu'ils utilisaient, malgré le décalage qui existe par moments entre leurs affirmations et les faits exposés.

La discussion des résultats met donc en relief de quelle façon les perceptions des participants sont appuyées par des faits qu'ils rapportent. Pour ce faire, nous avons croisé les récits avec les données sociodémographiques pour mettre en lien les perceptions exprimées par les participants avec certaines conditions de vie qu'ils ont connues depuis leur enfance. Nous avons également systématiquement comparé les mots qu'ils utilisaient pour qualifier leur enfance et les exemples qu'ils présentaient pour expliquer leur point de vue.

\section{Limites de la recherche}

Le nombre de participants que comporte la recherche ne permet pas de généraliser les résultats. D'autre part, il n'avait pas été prévu que l'on retrouverait dans plusieurs cas un décalage si important entre les qualificatifs utilisés par les participants par rapport à leur enfance et leur adolescence et les faits rapportés. II en découle une difficulté à les analyser de façon précise, car aucun outil n'a été prévu pour baliser cet aspect, ni de questions spécifiques dans le guide d'entrevue. L'analyse des données après coup présente donc cette limite, même si les résultats sont riches sur cette dimension de leur vie. 


\section{RÉsultats}

Les principales dimensions qui émergent du discours des jeunes hommes sont les liens avec leurs parents, leurs rapports avec le milieu scolaire et leur réseau social. Ces dimensions sont ici présentées telles que vécues durant l'enfance, puis l'adolescence.

\section{L'enfance}

Sur les 30 jeunes pères, 11 disent avoir connu une enfance heureuse, et leurs récits rapportent des moments agréables en famille, des rituels, le sentiment d'avoir été aimés de leurs parents.

Mon père, c'est mon frère t'sais? C'est mon meilleur ami, il a toujours été là pour moi, pour jouer avec moi, pour aller en bicycle, pour jouer au hockey, pour tout ce que tu veux... ma mère elle travaillait pas quand on était au primaire. C'était une mère au foyer parce qu'elle voulait vraiment prendre soin de nous autres. J'arrivais chez nous... j'mangeais pas à l'école avec les autres jeunes, genre, ma mère voulait me voir le midi. (Mathieu, 19 ans)

Lorsqu'ils la décrivent comme étant « passable », « correcte » (7), c'est que les moments heureux sont assombris par des déceptions, surtout la séparation des parents ou le sentiment d'avoir été abandonné par un de ceux-ci. Leurs propos sont souvent moins précis pour expliquer leurs perceptions :

Comme ci comme ça. Je n'ai pas beaucoup de mémoire de quand j'étais très jeune, mais il travaillait beaucoup. Fait que je le voyais plus ou moins souvent pis, un autre temps, on avait nos moments où on jouait ensemble pis on s'amusait, pis il y avait d'autres moments où on était plus à l'écart. (Didier, 22 ans)

Ils sont donc une légère majorité (18 sur 30) à estimer avoir connu une enfance heureuse ou « correcte », selon leurs propos.

Les jeunes hommes qui ont vécu une enfance difficile (7) ne le disent généralement pas comme tel, mais évoquent avoir vécu de la tristesse et relatent des événements qui les ont affectés. Ils s'expriment souvent de la façon suivante :

Mon père est parti d'chez nous quand j'avais 3 ans, fait qu'j'ai jamais connu mon père jusqu'à l'âge de 15 ans, pis j'ai trouvé ça dur à cause que, j'sais pas, comme j'voyais mes amis avec leur père, des choses de même, ça m'faisait comme d'la peine... (Donald, 15 ans)

Moi, ma mère avait pas vraiment l'temps, elle était, d'une façon... malgré qu'était tout l'temps avec nous autres, avait pas vraiment l'temps de s'occuper de deux enfants, faire la discipline avec les deux, y manquait quelqu'un, un pour un. (Marc, 23 ans)

Les participants qui relatent plus d'épisodes qui les ont affectés durant leur enfance ont tous vécu un sentiment d'abandon par un de leurs parents. Deux n'ont pas connu leur père, les autres ont subi les répercussions des séparations houleuses de leurs parents, particulièrement 
deux qui se sont sentis pris dans d'importants conflits entre eux. Trois ont éprouvé un profond sentiment d'avoir été abandonnés par leur mère ou leur père. Ils font tous état de l'instabilité qu'ils ont vécue : déménagements fréquents, changements de conjoints des parents, changement de garde entre leurs parents, négligence (un de ces jeunes a été placé en famille d'accueil par la Direction de la protection de la jeunesse).

Enfin, il y a les récits d'enfance où les interviewés relatent avoir vécu des événements violents (5), plaçant les enfants en situation de survie. Ceux que nous pourrions décrire comme des survivants de leur enfance ont tous été victimes de violence et témoins directs de violence à l'endroit de leur mère de la part de leur père ou d'un autre conjoint. Les situations classées dans cette catégorie sont sérieuses.

À la longue tu le sens pu. Tu pleures parce que ton humilité... te faire battre par ton père, mais tu sens pus les coups. T'es rendu que tu te les donnes toi-même... C'était pas à coups de claque mon père, c'était à coups de poing pis à coups de pied dans le corps pis il te garrochait en bas des marches. (Daniel, 24 ans)

Ces jeunes ont tous connu beaucoup d'instabilité, passant d'un lieu de résidence à un autre, de façon répétée et rapide, surtout au gré des changements de conjoint des parents.

\section{L'adolescence}

Chez la très grande majorité des jeunes hommes rencontrés, le passage à l'adolescence est trouble, faisant place à des comportements marginaux (qui nuisent à l'intégration sociale, telle une consommation abusive et régulière de substances), parfois délinquants (qui s'apparentent à la criminalité). D'abord, parmi les 11 qui estiment avoir connu une enfance heureuse, presque tous rapportent des comportements marginaux à l'adolescence. La moitié (6) ont consommé de l'alcool ou de la drogue à quelques reprises ou de façon répétée à l'école et quatre parmi eux ont développé une forte dépendance à une substance. Un autre a mentionné avoir eu de sérieux démêlés avec la justice pour des vols de voiture. Ils sont quatre à avoir été expulsés de l'école et un cinquième a pu l'éviter de justesse. Les jeunes de cette catégorie ont vécu en moyenne 5,4 déménagements durant leur enfance et leur adolescence (3,8 en enlevant une réponse extrêmement élevée). Enfin, la grande majorité de leurs parents étaient encore en couple au moment où ils ont appris qu'ils allaient devenir parents à leur tour (8 sur 11). Donc très peu d'entre eux ont connu d'autres figures parentales que leurs propres parents.

Parmi ceux qui estiment avoir connu une enfance «correcte » (7), certains ont également connu un passage houleux à l'adolescence. Ils ont rapporté des comportements marginaux ou délinquants, à différents degrés : consommation occasionnelle de drogue à l'école, défis très dangereux entre amis, batailles, vols, tentative de suicide. Deux des sept jeunes hommes de cette catégorie ont mentionné avoir développé une dépendance à la drogue. Deux ont mentionné avoir connu plusieurs difficultés dans leurs rapports avec leurs professeurs et la direction de leur école, avant de décrocher. Ils sont plus nombreux à avoir vécu des transitions familiales qui les amenaient à déménager souvent (5,9 fois, en moyenne). De plus, après la séparation de leurs parents, les jeunes hommes ont en moyenne connu 1,4 conjointe à leur père et 2,8 conjoints à leur mère. 
Pour leur part, les participants (7) ayant relaté davantage d'épisodes jugés difficiles durant leur enfance ont majoritairement connu un passage problématique à l'adolescence, où les comportements délinquants sont plus nombreux. Tous les jeunes de cette catégorie ont connu la séparation de leurs parents et n'ont pas eu le temps de s'enraciner dans un milieu, déménageant en moyenne 11,7 fois. À la suite de la séparation de leurs parents, ils ont connu en moyenne à leur père 2,4 conjointes et à leur mère, 4,1 conjoints.

Enfin, les participants (5) qui ont en quelque sorte survécu à leur enfance poursuivent une existence difficile à l'adolescence. Ils présentaient déjà au courant de leur enfance des comportements qui témoignaient de leur détresse et leur adolescence n'en est que la continuité. Quatre continuent de subir de la violence durant cette période. Ils refusent l'autorité des adultes dans tous les contextes. Cette catégorie comprend les jeunes les plus engagés dans la criminalité, sauf un, qui estimait avoir vécu une enfance acceptable. Trois sont devenus vendeurs de drogue avant 16 ans; un autre, sans en vendre, a commencé à prendre de la drogue à 11 ans et est devenu un très gros consommateur. II a également tenté de se suicider à quelques reprises. À l'opposé, l'un des cinq jeunes hommes, ayant souffert de l'alcoolisme de son père, a refusé toute consommation de drogue et d'alcool jusqu'à ce qu'il atteigne la vingtaine. Après la séparation de leurs parents, ces jeunes hommes ont vécu en moyenne 7,4 déménagements. Ils ont connu à leur père 1,6 conjointe et à leur mère, 7,2 conjoints en moyenne. Ces facteurs de risque sont supérieurs aux moyennes de l'échantillon global, présentées au tableau I : données sociodémographiques.

\section{INTERPRÉTATION DES RÉSULTATS}

La discussion portera d'abord sur les liens entre l'enfance, l'adolescence et les comportements marginaux des participants. Nous discuterons également du passage des jeunes pères vers la vie parentale et de leurs liens avec leurs propres parents. Enfin, nous discuterons des défis que présente l'analyse des points de vue des jeunes pères sur leur passé en relation avec les événements rapportés. Si la présentation des résultats a requis une fidélité aux propos tels que rapportés, la discussion qui suit nécessite une deuxième présentation, cette fois analytique, des résultats pour en dégager des tendances. Enfin, des repères théoriques pertinents à l'analyse des récits des jeunes pères seront proposés.

\section{Enfance, adolescence et comportements marginaux}

L'ensemble du portrait de l'enfance et de l'adolescence des jeunes pères qui ont participé à la recherche rejoint tout à fait celui qu'on brosse dans les écrits. Jusqu'à ce qu'ils apprennent qu'ils allaient devenir pères, ils avaient connu une adolescence tumultueuse dans une très importante proportion. La moyenne d'âge à laquelle les jeunes hommes disent avoir eu leur première relation sexuelle (15 ans et un mois) est plus basse que la moyenne canadienne qui est de 16 ans et demi (Rotterman, 2008). Cet âge ne semble pas être influencé par le type d'enfance qu'ils ont connu, sauf pour ceux qui ont vécu dans un contexte hostile. En effet, ces derniers déclarent avoir eu une première relation sexuelle en moyenne à l'âge de 13 ans. À l'instar de ce que constatent d'autres recherches, ils ont souvent consommé régulièrement différentes substances et certains en ont développé une dépendance (Dearden, Hale et 
Woolley, 1998). Également, ils ont démontré des tendances à la délinquance et, parfois, ont connu des démêlés avec la justice (Stouthamer-Loeber et Wei, 1998).

Appuyant ce que d'autres travaux ont relevé, le décrochage scolaire est le lot d'une proportion importante de l'échantillon, la moyenne de la dernière année scolaire réussie étant la $3^{\mathrm{e}}$ secondaire (Kiselica, 1999). Seulement quatre jeunes hommes possèdent un diplôme d'études secondaires, ce qui confirme l'important taux d'abandon scolaire chez les jeunes hommes qui vont devenir pères (Dudley et Stone, 2001). Toutefois, ce taux d'abandon varie entre les quatre catégories que nous avons dressées. Ceux qui persévèrent le plus sur le plan scolaire se retrouvent parmi ceux qui disent avoir connu une enfance heureuse (la moyenne de la dernière année scolaire réussie se situant pour cette catégorie à secondaire 3,8 ). Ceux qui rapportent avoir vécu une enfance « correcte » présentent un niveau de scolarité moyen plus faible que ceux qui déclarent avoir eu une enfance heureuse (secondaire 2,4). Les participants dont l'enfance fut décrite comme ayant été difficile ont malgré tout décroché un peu plus tard que ceux dont l'enfance était perçue comme acceptable ( $3^{\mathrm{e}}$ secondaire). Néanmoins, ils ont délaissé leur cheminement scolaire après avoir connu des rapports très conflictuels avec l'école. Pour ce qui est du sous-groupe que nous avons appelé des survivants, leurs rapports avec l'école, et la société en général, furent également très conflictuels. Ils ont décroché en moyenne en n'ayant obtenu qu'une $2^{\mathrm{e}}$ secondaire.

Le passage des jeunes pères vers la vie parentale et leurs liens avec leurs propres parents

Ces jeunes, qui sont en train de s'affranchir de leur rôle d'adolescent, éprouvent encore les besoins associés à leur groupe d'âge, en même temps qu'ils ont des besoins comme nouveaux parents. Ce contexte constitue un difficile passage pour leurs parents, qui deviennent grandsparents.

Nos résultats vont partiellement dans le sens des écrits sur le sujet qui rapportent que les jeunes pères ont habituellement peu de soutien de la part de leurs parents (Bunting et McAuley, 2004; Marsiglio, 1987). Ainsi, plusieurs jeunes pères ont souffert de l'absence de liens avec leur père ou ont connu des liens conflictuels avec celui-ci (Allen et Doherty, 1998). Toutefois, les écrits sur les jeunes pères font moins état de l'absence de la mère ou de profonds conflits avec elle, ce que nous retrouvons parmi les participants à notre étude. Cela dit, ces difficultés semblent servir de moteur d'engagement face à l'enfant naissant. Sortant eux-mêmes à peine de l'adolescence, les manques qu'ils ont vécus stimulent leur désir de faire mieux. En effet, le désengagement qu'ils ont observé chez leurs propres pères constitue parfois une source de motivation pour faire différemment et s'engager. Plusieurs veulent démontrer qu'ils peuvent faire mieux que leur propre père avec qui ils ont souvent connu une relation difficile.

Le vécu des jeunes pères avec leur père durant l'enfance façonne leurs perceptions du type de père qu'ils devraient être avec leur propre enfant. La souffrance liée à cette absence peut avoir nourri leur détermination de demeurer engagés auprès de leur enfant, d'être présents (Allen et Doherty, 1998, p. 156, traduction libre). 
Toutefois, ce constat requiert des nuances importantes. En effet, le nombre de participants à ne pas avoir de lien avec au moins un de leurs parents est important, mais le niveau de soutien provenant du parent avec qui ils sont en lien semble appréciable.

Le passage du rôle d'enfant qu'ils jouaient par rapport à leurs parents à celui de parents à leur tour, amène un regard nouveau sur leur vie, leurs comportements, de même que sur les façons dont leurs parents les ont éduqués. Ils saisissent davantage la complexité du rôle de parent et, quand le lien avec leurs parents était existant et significatif, il s'en trouve parfois amélioré. Toutefois, l'arrivée de leur enfant ne ravive pas un lien rompu avec leurs parents.

\section{Influence de l'adolescence sur l'expérience de la paternité précoce}

Ces jeunes hommes qui ont eu un enfant avec une jeune femme de moins de 20 ans rapportent une expérience du passage vers l'exercice de la paternité qui est différente de l'idée que l'on s'en fait. D'abord, à partir de nos données, le lien de causalité entre une enfance difficile et un passage difficile vers la vie adulte est ténu sous différents aspects: psychosocial, conjugal, socioéconomique et de l'engagement paternel.

Sur le plan psychosocial, malgré toutes les lacunes que l'on pourrait présumer retrouver chez les jeunes pères, l'ensemble des participants à la recherche, sauf quelques exceptions, rapportent s'adapter aux changements entraînés par leur nouveau rôle. Ils donnent de très nombreux exemples pour illustrer leurs propos. En fait, ils passent à l'âge adulte en même temps qu'ils endossent le rôle de père. Malgré les défis évidents de cette transition, leur expérience prend un sens pour eux d'une façon ou d'une autre, à des intensités différentes. Même s'il est difficile de vérifier à quel point ce sens s'ancre dans la réalité, malgré leur passé, nos résultats rejoignent ceux d'autres chercheurs : la paternité devient graduellement, en cours de grossesse, un projet (Reeves, 2006). Le sens qu'ils donnent à leur expérience constitue un important tampon entre les tensions liées au passage rapide, voire à une superposition des étapes de développement. Notamment, le sentiment d'améliorer sa vie, d'avoir des objectifs pour jouer son rôle auprès de son enfant, est une composante du sens de l'expérience des jeunes pères rencontrés. Cet aspect est de plus en plus reconnu par la recherche (Wilkinson, Magora, Garcia et Khurana, 2009; Ouellet, Milcent et Devault, 2006).

Sur le plan conjugal, la durée de la relation avec la jeune mère semble avoir plus d'influence sur le niveau de satisfaction des participants à l'égard de leur vie de couple que leur passé familial. Et même dans le cas où la relation est très récente, leur compagne occupe une place significative dans leur vie. Malgré les lacunes qu'ils peuvent présenter sur le plan des habiletés sociales, leur relation avec elle est perçue comme une partie importante de leur vie de père, même après une séparation (Deslauriers, 2010; Parra-Cardona, Sharp et Wampler, 2008). Rappelons qu'alors que leur enfant est âgé de 1 an, 22 jeunes pères sur 30 sont en couple avec la mère de leur enfant.

Sur le plan socioéconomique, tel qu'indiqué plus haut, un lien peut être établi entre leur vie familiale au cours de l'enfance et de l'adolescence et leur réussite scolaire. En outre, de façon corrélative, ceux qui estiment avoir connu une enfance heureuse ont en moyenne un revenu 
nettement plus élevé que les autres (voir tableau III). Toutefois, le lien entre l'enfance et le revenu n'est pas toujours celui que l'on présume : ceux dont l'enfance semble avoir été difficile ou une situation de survie ont des revenus plus importants que ceux qui qualifient leur enfance de « correcte ».

Enfin, concernant l'engagement paternel des jeunes pères à l'égard de leur enfant, encore ici nos résultats montrent que plusieurs jeunes ayant vécu des difficultés familiales importantes sont présents auprès de leur enfant et avaient maintenu un mode de vie stable un an après la naissance de leur enfant. Nombre d'auteurs rapportent que les jeunes pères sont beaucoup plus présents dans la vie de leur enfant que le veulent les idées qui circulent à leur sujet (Glikman, 2004). Et si on croise les données sociodémographiques avec les types d'enfance et d'adolescence que disent avoir connus les participants, on constate que ceux dont les enfances furent plus rudes sont proportionnellement plus nombreux à vivre sous le même toit que leur conjointe et leur enfant. Leur présence auprès de leur enfant est quotidienne, ce qui constitue un facteur crucial d'engagement paternel (Dudley, 2007). Nous n'avons pas d'information indiquant la qualité de leurs interactions, mais nous constatons ici que les difficultés connues dans l'enfance n'ont pas d'effet direct sur l'engagement des jeunes pères à l'égard de leur enfant un an après sa naissance. Ces résultats concordent avec ceux d'autres recherches qui démontrent que, malgré, ou en raison des expériences familiales difficiles, beaucoup de jeunes pères sont déterminés à devenir de bons pères (Devault, Milcent et Ouellet, 2005).

TABLEAU III

Type d'enfance et situation socioéconomique

\begin{tabular}{|l|c|c|}
\hline $\begin{array}{l}\text { Catégorie d'enfance telle que } \\
\text { présentée par les participants }\end{array}$ & $\begin{array}{l}\text { En couple avec la mère } \\
\text { de leur enfant un an } \\
\text { après la naissance }\end{array}$ & Revenu \\
\hline Heureuse & $6 / 11$ & 2126 \$ par mois \\
\hline « Correcte » & $5 / 7$ & 993 \$ par mois \\
\hline Difficile & $7 / 7$ & 1734 \$ par mois \\
\hline Survie & $4 / 5$ & 1455 \$ par mois \\
\hline
\end{tabular}

Enjeux et défis présentés par l'analyse du discours de jeunes pères

Tel qu'on l'a relevé plus tôt dans le présent article, le décalage entre le bilan que de jeunes pères font de leur passé et les exemples qu'ils rapportent présente un défi sur le plan de la compréhension que l'on peut en dégager. Avant de nous attarder à ce paradoxe, nous examinons ici les énoncés des participants qui présentent plus de cohésion avec les situations de vie objectives qu'ils ont décrites.

L'étude des témoignages des jeunes pères croisés avec certaines données sociodémographiques indique que les données se rejoignent lorsque nous mettons en lien le type d'enfance que les jeunes pères disent avoir connu et les transitions familiales vécues. Celles-ci constituent des repères qui aident à comprendre la trajectoire de vie des participants. 
Trois types de transitions familiales rapportées par les participants ont été compilés: les déménagements, les changements de conjoints de leur mère et les changements de conjointes de leur père.

TABLEAU IV

Transitions familiales et perceptions de l'enfance

\begin{tabular}{|l|c|c|c|}
\hline & Déménagements & $\begin{array}{l}\text { Nouveaux conjoints de } \\
\text { la mère }\end{array}$ & $\begin{array}{l}\text { Nouvelles conjointes } \\
\text { du père }\end{array}$ \\
\hline Heureuse & 5,4 & 1,2 & 0,7 \\
\hline «Correcte » & 5,9 & 2,8 & 1,4 \\
\hline Difficile & 11,7 & 4,1 & 2,4 \\
\hline Survie & 7,4 & 7,2 & 1,6 \\
\hline
\end{tabular}

On observe une concordance entre l'appréciation de l'enfance et les transitions familiales documentées. En effet, plus les jeunes pères affirment avoir connu une enfance heureuse, moins ils ont vécu de déménagements et moins ils ont connu de conjoints à leur père et à leur mère. À l'inverse, ceux qui ont connu une enfance difficile sont aussi ceux qui ont dû s'adapter à plusieurs déménagements et à de plus nombreux conjoints de la mère et conjointes du père.

Toutefois, le regard des participants sur leur enfance ne suffit pas toujours à prendre la mesure du bien-être ou des difficultés vécues au cours de cette étape de leur vie. De nombreux exemples de comportements marginaux rapportés par des jeunes qui ont décrit leur enfance comme étant heureuse ou acceptable apportent un autre éclairage sur leur trajectoire. En effet, seulement 3 des 30 participants n'ont pas relaté des difficultés majeures d'intégration sociale. Vingt-sept ont rapporté au moins un problème ou comportement parmi les quatre suivants: expulsion de l'école, dépendance à une substance, batailles à l'école et tentative de suicide.

On relève parfois une banalisation des événements chez certains. Les formules toutes faites comme «Tout s'est bien passé », " Ça n'a pas été un drame », "C'était correct » passent parfois sous silence des drames d'enfance ou d'adolescence. En effet, certains de ceux qui rapportent avoir connu une enfance «correcte » ont connu des épisodes plus éprouvants que ce qu'ils laissent entendre de prime abord. D'autres participants sont davantage en mesure d'expliquer les événements qui ont influencé leur vie, même s'ils éveillent des sentiments douloureux. Dans leur cas, un recul semble avoir été pris, de sorte qu'ils mesurent plus justement l'impact qu'ont eu ces événements sur leur vie. La cohérence du discours et le niveau de conscience face au passé varient donc d'un participant à un autre, rendant ardue l'analyse.

Le manque d'encadrement ou les drames de l'enfance, dont les conséquences ne s'étaient pas toujours fait sentir jusqu'à cette étape, apparaissent au grand jour à l'adolescence. Tel que mentionné plus tôt, les comportements marginaux sont très fréquemment rapportés par les participants lorsqu'ils parlent de leur adolescence, même quand ils posent un regard relativement positif sur leur enfance. Ce constat spontané en cours de cueillette de données nous a amenés à aborder davantage l'aspect de la dynamique familiale et plus particulièrement 
l'encadrement qu'ils ont connu dans leur famille d'origine. II s'est avéré que peu, parmi les 30 sujets, ont bénéficié d'un encadrement suffisamment structuré pour les aider à connaître les limites de leur liberté d'action dans leur famille et en société. Par exemple, certains mentionnent qu'ils « disaient tout » à l'un de leurs parents, le plus souvent la mère, ou aux deux au sujet de leur consommation de drogue. Dans la même lignée, les absences ou les difficultés à l'école n'étaient pas nécessairement considérées comme un problème par leurs parents. Un décalage survient donc parfois entre leur discours et les conditions objectives qui existaient durant leur adolescence. Un exemple typique : un futur jeune père qui n'a pas terminé sa $3^{\mathrm{e}}$ secondaire parce qu'il consommait de la marijuana tous les jours et qu'il avait été suspendu raconte ainsi son adolescence qu'il estime heureuse :

Y m'ont tout le temps encouragé, tout le temps aidé quand j'avais besoin de quoi. J'ai jamais eu la vie dure, mettons. J'ai jamais eu de gros obstacles durant ma vie, tout s'est bien passé. (Simon, 16 ans)

D'autres mentionnent que leurs comportements n'amenaient pas vraiment de réactions de la part de leurs parents qui n'exerçaient pas leur autorité :

Mon père, c'était mon meilleur ami, mais quand venait le temps d'être père, d'être sévère, il l'avait moins. Il était tout le temps là quand c'était le temps de jouer puis il a toujours été là, mais quand c'était le temps de finir, puis mettre les points, il a moins forcé là-dessus. (Mario, 17 ans)

Donc ces jeunes qui portent un regard neutre ou positif sur leur enfance et qui décrivent un lien positif avec un de leurs parents ou les deux ont néanmoins développé des schèmes de référence, des modes de vie et des comportements marginaux à divers degrés. II semble, comme d'autres études le rapportent, que ce n'est pas faute d'avoir bénéficié d'un lien très significatif avec au moins un de leurs parents, mais faute d'avoir été guidé de façon plus structurée, ce qui a nuit à l'établissement d'un lien sécurisant (Wallis et Steele, 2001).

D'autre part, le ton relativement neutre utilisé par les participants lorsqu'ils évoquaient leurs liens avec leurs parents a rendu moins apparente la souffrance qui a pu être vécue à ce propos. En révisant le contenu des entrevues, nous avons réalisé que sur les 30 jeunes hommes, 11 n'avaient jamais tissé de lien véritable ou avait rompu celui-ci, avec un de leurs parents (avec la mère dans 6 situations et avec le père dans 5 situations), ou même avec les deux dans un cas.

Le décalage entre les propos et les faits rapportés est plus fréquent chez les participants qui disent avoir connu une enfance heureuse ou satisfaisante. Chez ceux dont le discours laisse entrevoir une enfance difficile ou de survie, les faits rapportés concordent davantage avec leurs paroles. Même s'ils peuvent minimiser les difficultés vécues, il est plus facile d'établir un lien entre le type d'enfance connu, leurs propos à ce sujet et leur trajectoire de vie. Le lien entre l'expérience de l'enfance et les conduites marginales à l'adolescence est plus clair pour ceux qui rapportent avoir vécu plus de tristesse (7). Ceux-ci, en plus de ne pas avoir connu d'encadrement, ont souffert de la faiblesse ou de l'absence d'un lien d'attachement avec au moins un de leurs parents. Également, ils ont connu beaucoup de solitude, vivant seuls non seulement leurs problèmes familiaux, mais aussi leurs difficultés dans les autres sphères de 
leur vie. De plus, parmi eux se retrouvent des jeunes qui ont vécu des conflits avec les nouveaux conjoint(e)s de leur mère ou de leur père :

Mon père, y'était un gros manque dans ma vie. [...] Quand ma mère avait un chum, y voulait rien savoir de moi. C'était tout le temps : « Ah, j'ai pas le temps, va jouer dehors. » Ma mère a eu des chums qu'eux autres, c'était elle qu'y voulaient, puis moi, j'étais un fardeau. (Robert, 18 ans)

Ces participants expliquent qu'ils ont exprimé leur colère ou leur douleur par une révolte lors de l'adolescence, qui s'est souvent manifestée à l'école. L'intensité de cette rébellion est plus grande encore chez ceux qui ont vécu un sentiment d'abandon, des situations traumatisantes et de mauvais traitements durant leur enfance et leur adolescence, que ce soit d'un parent ou des conjoint(e)s de celui-ci.

Quelques repères théoriques pertinents à l'analyse du discours des jeunes pères sur leur passé Pour répondre aux défis particuliers que pose l'analyse de récits d'adolescents ou de jeunes adultes, deux repères théoriques s'avèrent pertinents : la socialisation de genre et la théorie de l'attachement (Bowlby, 1982), plus spécifiquement l'Entrevue sur l'attachement à l'âge adulte (Adult Attachment Interview de Main et Goldwin, 1985).

Depuis les années 1980, un grand nombre de recherches ont démontré que la socialisation masculine inhibe l'introspection et l'expression d'émotions (Tremblay, 2011. Ce champ de recherche a mené à étudier la façon dont les hommes font face à leurs difficultés personnelles (Dulac, 2001). On constate que l'expression de faiblesse ou de vulnérabilité constitue un échec, et par là un tabou, aux yeux de nombreux hommes, même chez les plus jeunes. Le dévoilement de difficultés familiales ou de situations abusives durant l'enfance s'en trouve donc limité, voire empêché (Dorais, 1997). Il n'est donc pas étonnant que de jeunes hommes parlent de difficultés personnelles en les minimisant, laissant entrevoir un écart entre les mots utilisés et la réalité objective. Ce phénomène est maintenant bien documenté en intervention sociale et est aussi à l'œuvre dans un contexte de recherche auprès d'une population masculine. L'interprétation du discours d'hommes de tous âges requiert donc une grande vigilance et doit tenir compte d'un certain filtre imposé par un schème de référence masculin dans la façon de se confier sur le plan personnel (Butera, 2006; Deslauriers et Deslauriers, 2011).

Bien que le concept de la socialisation masculine constitue un repère essentiel à l'analyse de récits d'hommes, il est insuffisant pour expliquer cet écart entre le regard que les jeunes hommes rencontrés portent sur leur vie et les explications données pour appuyer leurs dires. En effet, beaucoup de passages d'entrevues qui présentent des incongruités traitent des liens qu'ils ont avec leurs parents. Par exemple, certains mentionnent qu'ils avaient des liens très étroits avec leur mère sans être en mesure de donner des exemples de cette complicité. Les façons d'évoquer ou de décrire la qualité de leurs rapports avec leurs parents alors qu'ils étaient des consommateurs réguliers de drogues à l'école au su de leurs parents constituent un autre exemple. L'ambigüité qui peut se présenter dans l'interprétation de ce qu'est la « qualité » d'un lien décrit ainsi est plus difficile à dissiper à partir du concept de socialisation de genre. 
Nous estimons que l'utilisation de repères théoriques qui portent de façon spécifique sur les liens, tels que la théorie de l'attachement, est susceptible d'apporter un éclairage pertinent à l'analyse de discours. De façon plus spécifique, l'Entrevue sur l'attachement à l'âge adulte pourrait être utilisée (Bernier, Larose et Boivin, 1996). Cet outil permet de déterminer les types d'attachement vécus à l'âge adulte en lien avec ceux connus durant l'enfance: sécure/autonome, insécure/détaché ou évitant, insécure/préoccupé, insécure nonrésolu/désorganisé. Cette classification représente une continuité des types d'attachement élaborés par Ainsworth et ses collaborateurs (1978) suite à l'expérience de la Situation étrangère chez les enfants. Cette entrevue, visant à évaluer la capacité de l'adulte à assimiler des épisodes spécifiques de son enfance à une vision plus globale des relations parentsenfants, permet d'apprécier les mécanismes cognitifs déployés par le répondant pour traiter ces expériences (Bernier, Larose et Boivin, 1996). La cohérence de l'ensemble du discours, « et plus particulièrement, celle entre les adjectifs donnés pour chacun des parents et les exemples fournis pour les illustrer »(Guedeney et Guedeney, 2006, p. 130), demeure le principal indicateur de la sécurité de l'attachement. Également, la fluidité du discours et la capacité de l'illustrer de souvenirs heureux ou malheureux sont un indicateur de cohérence, et, par conséquent, d'un état d'esprit actuel sécure/autonome (Frodi et al., 2001, dans Théberge, 2009).

Néanmoins, même une méthode d'analyse plus systématique parvient difficilement à saisir à la fois les facteurs individuels, familiaux, culturels, sociaux et économiques qui influencent la trajectoire des individus. Également, même si on capte tous ces facteurs dans une analyse, mettre en lien le passé familial de jeunes pères avec leur transition vers la vie de parent et en établir la dynamique nécessite un devis de recherche pouvant rendre compte de la complexité de plusieurs variables objectives et subjectives. Le fait que des jeunes hommes ayant connu nombre de situations difficiles au cours de leur vie présentent une situation plus stable que prévu en est un exemple. Leur passé demeure un aspect crucial à considérer pour comprendre leur situation, et surtout le sens qu'ils y donnent, mais non comme un déterminant de réussite ou de risque, ni d'absence ou de présence dans la vie de leur enfant.

\section{Conclusion}

Le fait de connaître la trajectoire de vie des jeunes pères aide à comprendre de quelle façon l'avènement de la paternité s'y inscrit. Documenter les perceptions que les jeunes pères ont sur leur enfance et leur adolescence s'avère donc utile en recherche comme en intervention sociale. Par ailleurs, les résultats de cette recherche indiquent qu'un défi se pose concernant l'utilisation de cette information : celui du décalage qui existe souvent entre l'appréciation que de jeunes hommes font de leur passé et les faits qu'ils rapportent. Également, cette contradiction peut susciter un certain dilemme: aller dans le sens de ce que les jeunes perçoivent comme étant « correct », « normal » ou sonder la souffrance ou les carences qui ont pu être vécues lors de ces périodes, de même que les facteurs de risque que ces expériences représentent dans l'exercice de leur paternité. Cette rupture entre le regard que les jeunes pères posent sur leur enfance et le récit qu'ils en font mérite d'être étudiée afin de la comprendre et de trouver des pistes d'intervention pour aider ceux qui ont un passé chargé. 
Également, deux perspectives peuvent s'opposer en ce qui concerne l'intervention auprès des jeunes pères. D'une part, une approche basée sur la perspective des jeunes pères, des « cibles d'intervention » (que l'on « vise » très peu d'ailleurs), et d'autre part, une approche basée sur celle des « adultes », c'est-à-dire des intervenants et des chercheurs auxquels leur statut social confère l'autorité de déterminer ce qui est la norme en matière de parentalité. La tendance de ces derniers est souvent d'estimer que la transition des jeunes pères vers l'âge adulte est un échec! D'autant plus que les signes de difficultés sont présents. Notamment, ces jeunes pères ont presque tous présenté des «symptômes » de marginalité et n'ont que leur $3^{\mathrm{e}}$ secondaire en moyenne. Dans ce contexte, est-ce que les «adultes » considèrent comme valable le point de vue que les jeunes pères ont sur leur enfance? Font-ils l'équation entre enfance et adolescence difficiles et paternité à risque? Cette équation peut-elle contribuer à créer une réalité? À tout le moins, les possibilités d'intervention s'en trouvent diminuées.

De la même façon, il est crucial de trouver un dosage entre l'importance d'être à l'affût des facteurs de vulnérabilité, et celle de reconnaître les gestes que les jeunes pères posent pour passer de l'étape d'adolescent, ou de très jeune adulte, à celle de père. Serait-il possible de prendre en considération certaines forces présentes chez les jeunes pères et de miser sur elles, tout en reconnaissant des difficultés, des défis particuliers? Notamment, en examinant les données sociodémographiques, on voit bien qu'ils ne sont pas les déserteurs que l'on croit. Peut-on considérer ces changements effectués dans leur vie comme des indicateurs du désir d'assurer un certain bien-être à leur enfant sans oublier qu'ils demeurent pauvres et en situation de précarité? Est-il possible de percevoir que leur union amoureuse est plus significative que ce que l'on croit pour ces jeunes hommes, tout en reconnaissant la fragilité de leur union? Également, sommes-nous en mesure de reconnaître le sens qu'ils donnent à leur engagement auprès de leur enfant, tout en étant à l'affût des risques de désengagement dans un contexte de vulnérabilité sociale? Sur le terrain comme dans les milieux de recherche, on doit donner davantage la parole aux jeunes pères pour recueillir leurs perceptions et répondre à ces questions.

Jean-Martin Deslauriers

Professeur

École de service social Université d'Ottawa 


\section{BIBLIOGRAPHIE}

Ainsworth, M.D.S., M.C. Blehar, E. Waters et S. Wall (1978). Patterns of attachment: A psychological study of the strange situation, Hillsdale (NJ), Lawrence Erlbaum.

Allen, W.D. et W.J. Doherty (1996). « The responsibilities of fatherhood as perceived by African American teenage fathers », Families in Society. The Journal of Contemporary Human Services, vol. 77, $n^{\circ}$ 3, p. 142-155.

Allen, W. D. et W.J. Doherty (1998). « Being there: The Perception of the Fatherhood Among a Group of African-American », dans H.I. McCubbin et al., Resiliency in African American families, Thousand Oaks, Sage Publications, «Resiliency in families series », vol. 3, p. 207244.

Allen, W.D. et W.J. Doherty (2004). « Being There: The Perception of Fatherhood Among a Group of African American Adolescent Fathers », The Prevention Researcher, vol. 11, $\mathrm{n}^{\circ} 4$, p. 6-9.

Anda, R.F., V.J. Felitti, D.P. Chapman, J.B. Croft, D.F. Williamson, J. Santelli, P.M. Dietz et J.S. Marks (2001). «Abused Boys, Battered Mothers, and Male Involvement in Teen Pregnancy », Pediatrics, vol. 107, n² 2, p. 19-36.

Bernier, A., S. Larose et M. Boivin (1996). «L'entrevue sur l'attachement à l'âge adulte: fondements théoriques, qualités métrologiques et champs d'application », Psychologie canadienne, $\mathrm{n}^{\circ} 37$, p. 229-240.

Bowlby, J. (1982). Attachment, New York, Éditions Basic Books.

Bunting, L. et C. McAuley (2004). « Research Review: Teenage pregnancy and parenthood: the role of fathers », Child and Family Social Work, vol. 9, n³, p. 295-303.

Butera, K.J. (2006). «Manhunt: The Challenge of Enticing Men to Participate in a Study on Friendship », Qualitative Inquiry, vol. 12, n 6, p. 1262-1282.

Capaldi, D.M., K.C. Pears, G.R. Patterson et L.D. Owen (2003). « Continuity of parenting practices across generations in an at-risk sample: A prospective comparison of direct and mediated associations », Journal of Abnormal Child Psychology, vol. 31, n² 2, p. 127-142.

Charbonneau, J. (1999). « La maternité adolescente », Réseau, vol. 30, nº 7, p. 14-19.

Dearden, K., C. Hale et J. Alvarez (1992). «The educational antecedents of teen fatherhood », The British Journal of Educational Psychology, vol. 62, n 1, p. 139-147.

Dearden, K., C. Hale et M. Blankson (1994). « Family structure, function, and the early transition to fatherhood in Great Britain: Identifying antecedents using longitudinal data », Journal of Marriage and Family, vol. 56, $n^{\circ} 4$, p. 844-852.

Dearden, K.A., C.B. Hale et T. Woolley (1998). «The antecedents of teen fatherhood: A retrospective case-control study of Great Britain youth », American Journal of Public Health, vol. $85, n^{\circ} 4$, p. 551-554. 
Deslauriers, J.-M., G. Tremblay, S. Genest-Dufault, D. Blanchette et J.-Y. Desgagnés (2011). Regards sur les hommes et les masculinités: théories et pratiques, Sillery, Presses de l'Université Laval.

Deslauriers, J.-P. et J.-M. Deslauriers (2011). « La recherche auprès des hommes : défis et enjeux », dans Deslauriers, J.-M., G. Tremblay, S. Genest-Dufault, D. Blanchette et J.-Y. Desgagnés, Regards sur les hommes et les masculinités: théories et pratiques, Sillery, Presses de l'Université Laval, p. 153-172.

Deslauriers, J.-M. (2010). « L'expérience des futurs jeunes pères durant la période prénatale », Reflets : revue d'intervention sociale et communautaire, vol. 16, $\mathrm{n}^{\circ} 1$, p. 63-100.

Dorais, M. (1997). Ça arrive aussi aux garçons, Montréal, VLB.

Dudley, R. et G. Stone (2001). Fathering at risk, New York (NY), Springer Publishing.

Dudley, J.R. (2007). « Helping Nonresidential Fathers: The Case for Teen and Adult Unmarried Fathers », Families in Society, vol. 88, n², p. 171-181.

Dulac, G. (2001). Aider les hommes... aussi, Montréal, VLB.

Featherstone, B. (2009). Contemporary Fathering: Theory, Policy and Practice, Bristol, Policy Press.

Frewin, K., K. Tuffin et G. Rouch (2007). « Managing Identity: Adolescent Fathers Talk About the Transition to Parenthood », New Zealand Journal of Psychology, vol. 36, $\mathrm{n}^{\circ}$ 3, p. 161167.

Frodi, A., M. Dernevik, A. Sepa, J. Philipson et M. Bragesjö (2001). « Current attachment representations of incarcerated offenders varying in degree of psychopathy », Attachment and Human Development, vol. 3, n 3, p. 269-283.

Furstenberg, F.F. et C.C. Weiss (2000). «Intergenerational transmission of fathering roles in at risk families », Marriage and Family Review, vol. 29, $\mathrm{n}^{\text {os }} 2-3$, p. 181-201.

Glikman, H. (2004). «Low-Income Young Fathers: Contexts, Connections, and Self », Social Work, vol. 49, $\mathrm{n}^{\circ} 2$, p. 195-206.

Goulet, C., et al. (2001). « Le point sur les mères adolescentes au Québec », Ruptures, revue transdisciplinaire en santé, vol. $8, n^{\circ} 2$, p. 21-34.

Guedeney, A. et N. Guedeney (2006). L'attachement, concepts et applications, $2^{\mathrm{e}}$ éd., Paris, Masson.

Hanson, S.L., D.R. Morrison et A.L. Ginsburg (1989). «The antecedents of teenage fatherhood », Demography, vol. 26, n 4, p. 579-596.

Hardy, J.B. et L.S. Zabin (1991). Adolescent pregnancy in an urban environment. Issues, Programs, and Evaluation, Boston (MA), University Press of America.

Horne, A. M. et M. Kiselica (1999). Handbook of counseling boys and adolescent males, New Jersey, Sage Publications. 
Jaffee, S.R., A. Caspi, T.E. Moffitt, A. Taylor et N. Dickson (2001). « Predicting early fatherhood and whether young fathers live with their children: Prospective findings and policy reconsiderations », Journal of Child Psychology and Psychiatry, vol. 42, n 6, p. 803-815.

Kiernan, K.E. (1992). «The impact of family disruption in childhood on transitions made in young adult life », Population Studies, vol. 46, $\mathrm{n}^{\circ} 2$, p. 213-234.

Kiselica, M. (2008). When boys become parents, New Brunswick (NJ), Rutgers University Press.

Kiselica, M.S. (1999). «Counseling teen fathers », dans A.M. Horne et M.S. Kiselica, Handbook of Counseling Boys and Adolescent Males, Thousand Oaks (CA), Sage Publications, p. 179198.

Main, M., et R. Goldwyn (1985/1991). Adult attachment scoring and classification system. Unpublished manuscript, University of California, Berkeley.

Marsiglio, W. (1987). « Adolescent fathers in the United States: Their initial living arrangements, marital experience and educational outcomes », Family Planning Perspectives, vol. 19, $n^{\circ} 6$, p. $240-251$.

Ministère de la santé et des services sociaux. (2004). Les Services intégrés en périnatalité et pour la petite enfance à l'intention des familles vivant en contexte de vulnérabilité, Québec.

Ouellet, F., M.P. Milcent et A. Devault (2006). « Jeunes pères vulnérables : trajectoires de vie et paternité », Nouvelles pratiques sociales, vol. 18, n² 2, p. 156-171.

Parra-Cardona, J.R., E. Sharp et R.S. Wampler (2008). " "Changing for my kid": Fatherhood experiences of Mexican-Origin teen fathers involved in the justice system », Journal of Marital and Family Therapy, vol. 34, nº 3, p. 369-387.

Pears, K.C., S.L. Pierce, et al. (2005). « The Timing of Entry into Fatherhood in Young, At-Risk Men », Journal of Marriage and Family, vol. 67, n² 2, p. 429-447.

Reeves, J. (2006). « Recklessness, rescue and responsibility: Young men tell their stories of the transition to fatherhood », Practice, vol. 18, $n^{\circ} 2$, p. 79-86.

Rhoden, J.L. et B.E. Robinson (1997). «Teen dads. A generative fathering perspective versus The deficit myth », dans A.J. Hawkins et D.C. Dollahite, Generative fathering. Beyond deficit perspectives, Thousand Oaks (CA), Sage Publications, p. 105-117.

Rotterman, M. (2008). «Tendances du comportement sexuel et de l'utilisation du condom à l'adolescence », dans Rapport sur la santé, Ottawa, Statistique Canada.

Sigle-Rushton, W. (2005). «Young Fatherhood and Subsequent Disadvantage in the United Kingdom », Journal of Marriage and Family, vol. 67, $n^{\circ} 3$, p. 735-753.

Stouthamer-Loeber, M. et E.H. Wei (1998). « The precursors of young fatherhood and its effect on delinquency of teenage males », Journal of Adolescent Health, vol. 22, n 1, p. 56-65. 
Tremblay, G. et P. L'Heureux (2011). « La genèse de la construction de l'identité masculine », dans Deslauriers, J.-M., G. Tremblay, S. Genest-Dufault, D. Blanchette et J.-Y. Desgagnés, Regards sur les hommes et les masculinités: théories et pratiques, Sillery, Presses de I'Université Laval, p. 115-152.

Thompson, S.D. et A. Walker (2004). «Satisfaction with parenting: A comparison between adolescent mothers et fathers », Sex Roles, vol. 50, nos 9-10, p. 677-687.

Thornberry, T.P., C.A. Smith et G.G. Howard (1997). «Risk factors for teenage fatherhood », Journal of Marriage and Family, vol. 59, $n^{0} 3$, p. 505-522.

Tuffin, K., G. Rouch et K. Frewin (2010). « Constructing adolescent fatherhood: Responsibilities and intergenerational repair », Culture, Health and Sexuality, vol. 12, n 5, p. 485-498.

Wallis, P. et H. Steele (2001). « Attachment representations in adolescence: Further evidence from psychiatric residential settings », Attachment and Human Development, vol. 3, $\mathrm{n}^{\circ} 3$, p. 259-268.

Wilkinson, D., A. Magora, M. Garcia et A. Khurana (2009). « Fathering at the margins of society: Reflections from young, minority, crime-involved fathers », Journal of Family Issues, vol. 30, $\mathrm{n}^{0} 7$, p. $945-967$. 